\title{
A RADIOATIVIDADE DO METEORITO MARÍLIA (QUEDA DO DIA 5 DE OUTUBRO DE 1971) MEDIDA POR ESPECTROMETRIA GAMA*
}

\author{
DANIEL NORDEMANN** e TEREZA SAKAI \\ Programa de Pesquisa e Pós-graduação em Geofísica, \\ Universidade Federal da Bahia
}

\begin{abstract}
The activities of the radionuclides $\mathrm{Mn}^{54}, \mathrm{Na}^{22}, \mathrm{Co}^{60}, \mathrm{Al}^{26}$ and $\mathrm{K}^{40}$ were measured by low background scintillation gamma-ray spectrometry on two specimen (106 g and $780 \mathrm{~g})$ of the Marilia stone meteorite of the fall of October 5, 1971 (Marília, State of São Paulo, Brazil). These activities are, except for potassium 40 , due to nuclear reactions induced by the cosmic-ray irradiation of the meteorite in space. From the interpretation of the activities measured, the following results were obtained:
\end{abstract}

$$
\begin{aligned}
& \text { potassium content: } 0.084 \% \pm 0.005 \% \\
& \text { mass of the meteorite in space } \geqslant 50 \mathrm{~kg} ; \\
& \text { age of cosmic-ray exposure } \geqslant 1.3 \times 10^{6} \text { years. }
\end{aligned}
$$

INTRODUC̣Ão No dia 5 de outubro de 1971, às 17 horas (tempo local), um meteorito de pedra (condrito) caiu em Marília (22 $\left.2^{\circ} 15^{\prime} \mathrm{S}, 49^{\circ} 56^{\prime} \mathrm{W}\right)$, no Estado de São Paulo, Brasil. Numerosos fragmentos do meteorito foram recolhidos numa área de aproximadamente $2 \mathrm{~km}^{2}$. Infelizmente poucos desses fragmentos, representando uma massa de aproximadamente $2,5 \mathrm{~kg}$, puderam chegar aos laboratórios ou museus, por causa da curiosidade popular e da vontade de ficar com uma lembrança desse acontecimento raro. Graças à boa vontade do professor Moacir Arruda, do Instituto de Geociências e Astronomia da Universidade de São Paulo e do professor Paulo Eduardo Avanzo, da Faculdade de Filosofia de Marília, tivemos um pedaço de $106 \mathrm{~g}$ doado e um de $780 \mathrm{~g}$ emprestado. Esses dois pedaços, de forma irregular, apresentam uma parte da sua superfície coberta por crosta de fusão feita durante a queda na atmosfera terrestre, o que confirma, com a extensão da zona da queda. uma fragmentação a uma altitude bastante elevada, da ordem de $15 \mathrm{~km}$ (Nordemann, Tobailem, de Lassus Saint-Genies, 1970).

Medidas de espectrometria gama de baixo ruído de fundo, de 1000 minutos cada uma, foram feitas sobre os dois pedaços, no laboratório de Geofísica Nuclear do Instituto de Física da Universidade Federal da Bahia. Trata-se de medidas não-destrutivas, o que é um fator muito importante quando as amostras são meteoritos que represéntam um material extraterrestre muito raro (o material mais raro até agora são as rochas da Lua). Os radionuclídeos emissores gama dos meteoritos são, na maioria, produtos de espalação feitos pelas reações nucleares induzidas pela radiação cósmica na matéria do próprio meteorito durante a sua trajetória interplanetária (Tab. I). Algumas informações típicas que esse tipo de medida permite obter são relativas ao tempo de irradiação cósmica, desde a ruptura de corpos maiores, possíveis geradores de asteróides e meteoritos, às variações da intensidade da radiação cósmica em função do tempo e do espaço, ao tempo entre a queda e as medidas (no caso de meteoritos achados).

*Trabalho apresentado na XXIV Reunião Anual da Sociedade Brasileira para o Progresso da Ciência; São Paulo, 1972

**Projeto BRA-562 do PNUD(SF) - UNESCO 
Tabela I - Principais emissores gama dos meteoritos de pedra, faixas de energia usadas e rendimentos de deteção.

\begin{tabular}{|c|c|c|c|c|c|c|}
\hline \multirow{2}{*}{ Nuclídeo } & \multirow{2}{*}{$\begin{array}{c}\text { Emissões } \\
\text { gama } \\
(\mathrm{MeV})\end{array}$} & \multirow{2}{*}{$\begin{array}{l}\text { Faixa } \\
\text { usada } \\
(\mathrm{MeV})\end{array}$} & \multirow{2}{*}{$\begin{array}{c}\text { Equação } \\
\text { correspondente }\end{array}$} & \multirow{2}{*}{$\begin{array}{c}\text { Contribuição } \\
\text { na faixa }\end{array}$} & \multicolumn{2}{|c|}{$\begin{array}{l}\text { Rendimentos } \\
\text { de deteção }\end{array}$} \\
\hline & & & & & $106 \mathrm{~g}$ & $780 \mathrm{~g}$ \\
\hline${ }^{54} \mathrm{Mn}$ & 0,835 & 0,$75 ; 0,90$ & (1) & $M$ & $8,30 \%$ & $5,00 \%$ \\
\hline${ }^{22} \mathrm{Na}$ & $\begin{array}{l}0,511\left(\beta^{+}\right) \\
1,280\end{array}$ & 1,$20 ; 1,34$ & (2) & $N$ & $3,53 \%$ & $2,22 \%$ \\
\hline${ }^{60} \mathrm{Ca}$ & $\begin{array}{l}1,170 \\
1,330\end{array}$ & 1,$11 ; 1,44$ & & & $9,52 \%$ & \\
\hline${ }^{40} \mathrm{~K}$ & 1,462 & 1,$35 ; 1,56$ & (3) & K & $0,40 \%$ & $0,25 \%$ \\
\hline${ }^{26} \mathrm{Al}$ & $\begin{array}{l}0,511\left(\beta^{+}\right) \\
1,830\end{array}$ & 1,$73 ; 1.95$ & (4) & A & $3.15 \%$ & $1,67 \%$ \\
\hline
\end{tabular}

A instalação de espectrometria gama usada compreende principalmente um detetor de cintilação com um cristal de iodeto de sódio ativado com tálio, de $10 \times 8 \mathrm{~cm}$, associado a uma fotomultiplicadora com janela de quartzo da "Quartz et Silice", um castelo de ferro (2,5 cm de espessura) e de chumbo (16 cm de espessura) de 10 toneladas, um analisador de pulsos da "Hewlett-Packard" de 1024 canais. O sistema foi regulado para ter $10 \mathrm{keV} /$ canal e a estabilidade é bastante boa para não haver aumento notável da resolução, para medidas dessa duração (1000 minutos).

Esse sistema de deteção é semelhante ao do laboratório Centre des Faibles Radioactivités, de Gif-sur-Yvette (França), o que nos permitiu usar os mesmos rendimentos de deteção determinados para os estudos dos meteoritos Dosso, num fragmento de $805 \mathrm{~g}$ (Tobailem, Nordemann, 1970), e Tillaberi, num fragmento de $146 \mathrm{~g}$ (rendimentos esses que nos foram cedidos através de uma amigável comunicação com Tobailem). Esses rendimentos foram obtidos a partir das medidas de espectrometria gama feita sobre maquetes de fontes radioativas padrão, simulando fragmentos de meteoritos de mesma natureza, sob as mesmas condições quanto ao tamanho, forma e geometria, de modo a reproduzir as mesmas condições de absorção da radiação gama pela própria fonte.

$\mathrm{O}$ uso desses rendimentos para os fragmentos do meteorito Marília foi possível porque correspondiam bastante a nossas condições de medida. Os erros prováveis devidos à imperfeição da simulação estão incluídos nas incertezas ligadas a esses rendimentos (Tab. I).

Sabe-se que a espectrometria gama permite identificar os vários emissores gama presentes em quantidade suficiente na amostra medida, graças aos picos de absorção total. Mas o efeito Compton faz com que a superficie compreendida dentro da faixa de energia que inclui o pico de absorção total, inclua também uma contribuição dos outros emissores gama de energia superior. Por isso, os cálculos de determinação das atividades individuais são feitos através da resolução de um sistema de equações cujos termos numéricos foram determinados graças às calibrações já mencionadas. $\mathrm{O}$ sistema usado inicialmente compreendia uma equação relativa à faixa do cobalto 60 , mas, devido à fraca atividade encontrada para esse nuclídeo, foi mais conveniente dar uma estimação superior do teor em cobalto 60 e usar os sistemas simplificados que apresentamos a seguir.

Caso do fragmento de $106 \mathrm{~g}$

$$
\begin{aligned}
M+0,55 N+0,27 K+0,40 A & =1072 \\
N+0,17 K+0,29 A & =462 \\
0,40 N+K+0,47 A & =820 \\
0,33 N+A & =235
\end{aligned}
$$


Caso do fragmento de $780 \mathrm{~g}$

$$
\begin{aligned}
M+0,50 N+0,32 K+0,69 A & =3845 \\
N+0,20 K+0,45 A & =1847 \\
0,18 N+K+0,47 A & =3548 \\
0,20 N+A & =838
\end{aligned}
$$

As contagens e as contribuições dos emissores nas várias faixas de energia são expressas em pulsos por 1000 minutos, pois as medidas foram feitas nessa duração. A Tab. I apresenta as principais emissões gama, as faixas escolhidas para a elaboração do sistema de equações, os símbolos da contribuição de cada nuclídeo em sua faixa principal e os rendimentos que foram usados para passar das contribuições $M, N, K$ e $A$ às atividades dos nuclídeos.

A Tab. II apresenta os resultados das medidas expressas em desintegrações por minuto e por quilograma de meteorito $\left(\mathrm{dpm} \cdot \mathrm{kg}^{-1}\right)$, no dia da queda, ao lado do período dos nuclídeos e de seus principais modos de formação. As incertezas incluem flutuações devidas às contagens realizadas (critério de um desvio-padrão $\sigma$ ), os erros sobre as calibrações (contagens e fontes-padrão) e um termo devido à imperfeição da simulação dos

\begin{tabular}{|c|c|c|c|c|c|}
\hline \multirow{2}{*}{ Nuclideos } & \multirow{2}{*}{ Períodos } & \multirow{2}{*}{$\begin{array}{l}\text { Principais } \\
\text { modos de } \\
\text { formação }\end{array}$} & Atividades & $\left(\mathrm{dpm} \cdot \mathrm{kg}^{-1}\right)$ & \multirow{2}{*}{$\begin{array}{l}\text { Relação das } \\
\text { atividades } \\
780 \mathrm{~g} / 106 \mathrm{~g}\end{array}$} \\
\hline & & & $106 \mathrm{~g}$ & $780 \mathrm{~g}$ & \\
\hline${ }^{54} \mathrm{Mn}$ & 311,9 dias & $\begin{array}{l}{ }^{56} \mathrm{Fe}(p, 2 p n){ }^{54} \mathrm{Mn} \\
{ }^{54} \mathrm{Fe}(n, p){ }^{54} \mathrm{Mn} \\
{ }^{56} \mathrm{Fe}(n, p 2 n)^{54} \mathrm{Mn}\end{array}$ & $108 \pm 25$ & $79 \pm 7$ & 0,72 \\
\hline${ }^{22} \mathrm{Na}$ & 2,60 anos & $\begin{array}{l}{ }^{24} \mathrm{Mg}(p, 2 p n)^{22} \mathrm{Na} \\
{ }^{28} \mathrm{Si}(p, 4 p 3 n)^{22} \mathrm{Na} \\
{ }^{23} \mathrm{Na}(n, 2 n)^{22} \mathrm{Na}\end{array}$ & $94 \pm 33$ & $62 \pm 7$ & 0,67 \\
\hline${ }^{60} \mathrm{Co}$ & 5,27 anos & ${ }^{59} \mathrm{Co}(n, \gamma){ }^{60} \mathrm{Co}$ & $20 \leqslant$ & $6 \leqslant$ & \\
\hline${ }^{26} \mathrm{Al}$ & $7,38 \cdot 10^{5}$ anos & ${ }^{28} \mathrm{Si}(p, 2 p n){ }^{26} \mathrm{Al}$ & $39 \pm 35$ & $50 \pm 12$ & 1,28 \\
\hline${ }^{40} \mathrm{~K}$ & $1,265 \cdot 10^{9}$ anos & Nucleossintese & $1490 \pm 200$ & $1570 \pm 80$ & 1,05 \\
\hline
\end{tabular}
fragmentos na realização das calibrações.

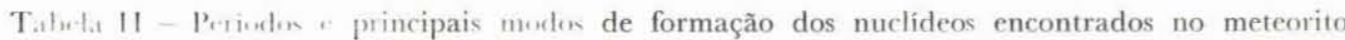
Marilia; resultados das medidas de espectrometria gama

Os resultados obtidos para cada fragmento são apresentados na Tab. II a fim de compará-los. Vale salientar que é possível encontrar valores diferentes nos nuclídeos formados pela ação da radiação cósmica em função da localização dos pedaços dentro do meteorito principal (antes de se quebrar na entrada na atmosfera), com excessão do potássio 40 .

O potássio 40 é resíduo da nucleossíntese e os teores devem se encontrar próximos devido a uma repartição bastante homogênea dentro do meteorito. Assim, os valores encontrados de $(1570 \pm 80) \mathrm{dpm} \cdot \mathrm{kg}^{-1}$ no fragmento de $780 \mathrm{~g}$ e de $(1490 \pm 200) \mathrm{dpm} \cdot \mathrm{kg}^{-1}$ no fragmento de $106 \mathrm{~g}$ são quase iguais. Como a composição isotópica do potássio nos meteoritos de pedra é idêntica à composição isotópica do potássio das rochas da Terra (uma vez que o potássio de origem cosmogênica é desprezível), podemos determinar o teor do potássio a partir da atividade do potássio 40. Usando a relação isotópica ${ }^{40} \mathrm{~K} / \mathrm{K}=0,0118 \%$ e a atividade do potássio $40\left(1570 \pm 80 \mathrm{dpm} \cdot \mathrm{kg}^{-1}\right)$, achamos um teor em potássio de $0,084 \% \pm 0,005 \%$. Esse valor está de acordo com os teores em potássio obtidos para outros meteoritos de pedra desse tipo. 
Nos outros nuclídeos, todos cosmogênicos, verificou-se que as atividades encontradas no fragmento de $780 \mathrm{~g}$ eram nitidamente mais fracas do que as encontradas no fragmento de $106 \mathrm{~g}$, indicando que a matéria do fragmento de $780 \mathrm{~g}$ foi localizada mais no interior do meteorito espacial do que a matéria do fragmento de $106 \mathrm{~g}$. Como o coeficiente médio de absorção da radiação cósmica é aproximadamente igual a $150 \mathrm{~g} \cdot \mathrm{cm}^{2}$ (Nordemann, 1966), é possível calcular, a partir da relação média das atividades dos dois pedaços (eliminando-se a relação das atividades do alumínio 26, cuja incerteza é muito grande), a profundidade do pedaço de $780 \mathrm{~g}$, com relação ao pedaço de $106 \mathrm{~g}$. Supondo a densidade média do meteorito igual a $3,5 \mathrm{~g} \cdot \mathrm{cm}^{-3}$ e o pedaço de $106 \mathrm{~g}$ localizado na superfície do meteorito no espaço, foi obtida uma profundidade de $15 \mathrm{~cm}$ para o pedaço de $780 \mathrm{~g}$, o que dá uma massa total mínima do meteorito espacial de $50 \mathrm{~kg}$.

Podemos tentar estimar a idade de exposição à radiação cósmica a partir da atividade do alumínio 26, usando a relação conhecida do aumento da atividade do nuclídeo radioativo gerado no espaço pela radiação cósmica (Heymann, Anders, 1967):

$$
{ }^{26} \mathrm{Al}=\left({ }^{26} \mathrm{Al}\right)_{s}\left(1-e^{-\lambda t}\right),
$$

${ }^{26} \mathrm{Al}$ : atividade medida do alumínio 26 para um meteorito irradiado durante um tempo $t$ chamado idade de irradiação

$\left({ }^{26} \mathrm{Al}\right)_{s}$ : atividade à saturação do alumínio 26 para um meteorito da mesma composição química

$\lambda$ : $\quad$ constante radioativa do alumínio 26 , ligada ao período radioativo $T$ do alumínio 26 pela relação $\lambda=0,693 / T$.

Uma vez que o fragmento de $780 \mathrm{~g}$, provavelmente, estava localizado a uma certa profundidade dentro do meteorito espacial, não é possível considerar somente a atividade do alumínio 26 , pois essa atividade pode ser menor dentro do meteorito por causa da irradiação cósmica atenuada pela absorção e não apenas pela falta de saturação da atividade em função do tempo (Fuse, Anders, 1969). Então, usa-se a relação ${ }^{26} \mathrm{Al} /{ }^{22} \mathrm{Na}$ cujo valor é sensivelmente independente da profundidade num meteorito dado, por causa dos modos de formação comparáveis dos dois nuclídeos ${ }^{26} \mathrm{Al} \mathrm{e}{ }^{22} \mathrm{Na}$. Mas a atividade do sódio 22 de um período de 2,60 anos varia em função do tempo nos meteoritos no espaço por causa da modulação da radiação cósmica em relação à atividade solar. Isso faz com que, para os meteoritos de idade grande, em comparação com o período do alumínio $26\left(7,38 \cdot 10^{5}\right.$ anos), a relação ${ }^{26} \mathrm{Al} /{ }^{22} \mathrm{Na}$ ou ${ }^{22} \mathrm{Na} /{ }^{26} \mathrm{Al}$ varie em função da data de queda (Fireman, 1967; Tobailem, Nordemann, 1970; Tobailem, Lalou, 1972). A curva da relação ${ }^{22} \mathrm{Na} /{ }^{26} \mathrm{Al}$ em função da data da queda, apresentada no trabalho de Tobailem e Lalou (1972) permite obter o valor teórico da relação para um meteorito que teria caído em outubro dẹ 1971 e que teria atingido o valor de saturação para o alumínio 26. A relação achada na curva teórica é ${ }^{22} \mathrm{Na} /{ }^{26} \mathrm{Al}=1,2$, o que dá ${ }^{26} \mathrm{Al} /{ }^{22} \mathrm{Na}=0,83$ enquanto a relação medida para o pedaço de $780 \mathrm{~g}$ vale $0,81 \pm 0,22$. O valor da atividade do alumínio 26 é da ordem de grandeza do valor de saturação, indicando uma idade de exposição superior a $1,3 \cdot 10^{6}$ anos (levando-se em consideração a incerteza sobre a relação medida):

$$
T \text { irradiação } \geqslant 1,3 \cdot 10^{6} \text { anos. }
$$

Nesse caso, pode-se dizer, por exemplo, que o meteorito Marília tem uma idade de exposição à radiação cósmica superior à idade obtida de maneira comparável para o meteorito Bovedy: $(0,9 \pm 0,3) \cdot 10^{6}$ anos (Tobailem, Nordemann, Grjebine, 1971). 
Agradecimentos Agradecemos ao Dr. J. Tobailem do Centre des Faibles Radioactivités, C.N.R.S., Gif-sur-Yvette (França), e do Service d'Electronique Physique, C.E.N. de Saclay (França) pela comunicação dos rendimentos de deteção, e aos professores Moacir Arruda (Instituto de Geociências e Astronomia da Universidade de São Paulo) e Paulo Eduardo Avanzo (Faculdade de Filosofia de Marília), que cederam os pedaços do meteorito para nosso estudo.

\section{BIBLIOGRAFIA}

HEYMANN, D. e ANDERS, E. - 1967 - Meteorites with short cosmic-ray exposure ages, as determined from their Al 26 content. Geochim. Cosmochim. Acta, 31: 1793-1 809

FIREMAN, E. L. - 1967 - Radioactivities in meteorites and cosmic-ray variations. Geochim. Cosmochim. Acta, 31: 1691-1 700

FUSE, K. e ANDERS, E. - 1969 - Aluminum 26 in meteorites, VI. Geochim. Cosmochim. Acta, 33: $653-670$

NORDEMANN, D. - 1966 - Emissions gamma de quelques météorites et roches terrestres, évaluation de la radioactivité du sol lunaire. Tese, Paris (França) e Rapport CEA-R 3017 (França)

NORDEMANN, D., TOBAILEM, J. e de LASSUS SAINT-GENIES, Ch. H. - 1970 - La météorite Saint-Séverin, recherche de la trajectoire atmosphérique et de l'orbite. Rapport CEA-R 4045 (França)

TOBAIlEM, J. e LALOU, C. - 1972 - Age d'exposition de la météorite Kiffa. G. R. Acad. Sci. Paris (França), em preparação

TOBAILEM, J. e NORDEMANN, D. - 1970 - La radioactivité de la météorite Dosso mesurée par spectrométrie gamma. C. R. Acad. Sci. Paris (França), 271: 262-264

TOBAILEM, J., NORDEMANN, D. e GRJEBINE, T. - 1971 - Gamma emitters and exposure age of the Bovedy meteorite of the fall of April 25, 1969. Nature Physical Science, 229 (4): 118-119 